\title{
No Association between Preeclampsia or Cesarean Section and Incidence of Type 1 Diabetes among Children: A Large, Population-Based Cohort Study
}

\author{
LARS C. STENE, PER MAGNUS, ROLV T. LIE, ODDMUND S $\emptyset$ VIK, GEIR JONER, AND THE \\ NORWEGIAN CHILDHOOD DIABETES STUDY GROUP \\ Diabetes Research Centre, Aker and Ullevål Hospitals, Department of Paediatrics, Ullevål Hospital, \\ N-0407 Oslo, Norway [L.C.S., G.J.], Division of Epidemiology, Norwegian Institute of Public Health, \\ 0403 Oslo, Norway [L.C.S., P.M., G.J.], Medical Birth Registry of Norway and Section for Medical \\ Statistics, Department of Public Health and Primary Health Care, University of Bergen, N-5021 Bergen, \\ Norway [R.T.L.], and Department of Paediatrics, Haukeland Hospital, N-5021 Bergen, Norway [O.S.]
}

\section{ABSTRACT}

The objective was to investigate whether selected perinatal factors, as indicators of perinatal exposures, are associated with the risk of type 1 diabetes in children. Specifically, we tested whether maternal preeclampsia, Rhesus-immunization, induced labor, cesarean section, and multiple birth were associated with incidence of type 1 diabetes. A cohort study was designed by linking records of the Medical Birth Registry and the National Childhood Diabetes Registry of Norway. Live births in the study period were followed for a maximum of $15 \mathrm{y}$ and contributed 8,166,731 person-years between 1989 and 1998. Altogether, 1824 cases of type 1 diabetes diagnosed between 1989 and 1998 were identified within the cohort. There was a suggestive, but nonsignificant, increase in risk of type 1 diabetes associated with Rhesus-immunization. Maternal preeclampsia, cesarean section, and the other perinatal factors investigated in this study were not significantly associated with incidence of type 1 diabetes in the children. Previous indications that cesarean section and preeclampsia are associated with type 1 diabetes were not supported by this large study. The majority of routinely recorded perinatal factors are only weakly associated with type 1 diabetes, or not at all. (Pediatr Res 54: 487-490, 2003)
Type 1 diabetes mellitus is a consequence of an immunemediated destruction of the pancreatic $\beta$ cells, and is one of the most common chronic and life-long diseases in childhood. The factors initiating the destructive process are largely unknown, but genetic factors as well as nongenetic factors are involved (1). The relatively young age at onset and the long preclinical phase in type 1 diabetes suggest that environmental risk factors may play a role early in life, possibly in utero (2). Some perinatal factors, such as birth weight (3), birth order, and maternal age (4), and complications during pregnancy (5-9) may be taken as markers of various environmental exposures in utero or early in life. Environmental exposures of potential

Received December 30, 2002; accepted April 21, 2003.

Correspondence: Lars C. Stene, Division of Epidemiology, Norwegian Institute of Public Health, P.O. Box 4404 Nydalen, N-0403, Oslo, Norway; e-mail: lars.christian.stene@fhi.no

L.C.S. and G.J. were supported by a grant from the Norwegian Foundation for Health and Rehabilitation (grant no. 1997/156), a grant from The Research Council of Norway (148359/330), and grants from the Norwegian Diabetes Association. Funding was also provided by Tine Norwegian Dairies and Novo Nordisk Pharma A/S.

DOI: 10.1203/01.PDR.0000081301.25600.5D importance in the etiology of type 1 diabetes may be growth rate in utero or early life, timing of bacterial colonization as influenced by mode of delivery (10), or metabolic and immunologic events influenced by maternal age, birth order (4), mode of delivery (11), maternal preeclampsia, fetomaternal blood group incompatibility, or other maternal pregnancy complications. Previous case-control studies have found indications that preeclampsia $(5,7-9)$ and cesarean section $(5,6)$ are associated with increased risk of type 1 diabetes, but other studies have shown apparently inconsistent results $(6,9,12)$. Although the inconsistent findings may be due to a number of factors, the combination of relatively weak associations and small to moderate sample sizes may lead to variable results between studies because of sample variation. We have previously shown that a large study can demonstrate consistent and sometimes novel results, even when the associations are relatively weak $(3,4)$. The objective of the present study was to estimate the associations of maternal preeclampsia, Rhesusimmunization, induced labor, cesarean section, and multiple births with the incidence of type 1 diabetes in children, using a cohort design and a large sample size. 


\section{MATERIALS AND METHODS}

Subjects. In Norway, all newly diagnosed cases of type 1 diabetes before $15 \mathrm{y}$ of age have been prospectively registered in the National Childhood Diabetes Registry since January 1, 1989, using the EURODIAB diagnostic criteria (13). We designed a cohort study by record linkage of the Medical Birth Registry and the National Childhood Diabetes Registry via the unique personal identification number assigned to all residents of Norway $(3,4)$. The study was approved by the regional ethics committee and the National Data Inspectorate. The Medical Birth Registry of Norway has registered all births in Norway since 1967, and includes information on a number of perinatal variables $(14,15)$. Out of 1863 cases of type 1 diabetes diagnosed between January 1, 1989, and December 31, 1998, 1824 were linked. All live births in Norway between 1974 and 1998 contributed time under observation from birth to type 1 diabetes (from 1989 to 1998), death in the first year of life, age 15 y, or December 31, 1998, whichever occurred first. Because registration of cases started in 1989, time under observation was counted only from January 1, 1989, for those born before this date. This means that, even if some cases of type 1 diabetes may have occurred among those born between 1974 and 1989, it would not influence our results $(3,4,16)$. A total of $1,382,602$ individuals contributed $8,166,731$ personyears under observation between 1989 and 1998. The mean time from birth to type 1 diabetes or censoring was 10.2 y (SD $=5.0$ ), and the mean time under observation after January 1, 1989 , was 5.9 y $(\mathrm{SD}=3.3)$. The mean age at diagnosis among the 1824 who developed type 1 diabetes was 8.6 y $(\mathrm{SD}=3.7)$.

Variables. Maternal diabetes mellitus (any type) diagnosed before or during pregnancy, maternal preeclampsia, Rhesusimmunization, artificial induction of labor, cesarean delivery, twin or higher-order multiple birth, and sex as registered in the Medical Birth Registry were evaluated as possible risk factors. Individuals with missing data on birth weight or birth order
( $0.3 \%$ of the cohort, including 14 who developed type 1 diabetes) were excluded from analyses adjusted for these variables.

Statistical analysis. The number of incident cases and person-time under observation in each exposure category were computed using DATAB in the EPICURE package, version $1.8 \mathrm{w}$ (Hirosoft International Corporation, Seattle, WA, U.S.A.) (17). Rate ratios with $95 \%$ confidence intervals were estimated with Poisson regression analyses. We included sex, age group in 3-y categories, and calendar period of birth in 5-y categories to adjust for possible effects of those variables. Furthermore, maternal diabetes, birth weight (3), maternal age at delivery, birth order, and an interaction term between maternal age and birth order (4) were entered in the regression models. We further assessed possible confounding by differences in reporting or other systematic differences between geographical regions in Norway by adjusting for county of birth. There are 19 counties in Norway, each with a central hospital where the majority of deliveries takes place.

\section{RESULTS}

There was a tendency that Rhesus-immunization was associated with increased incidence of type 1 diabetes, but the confidence interval was relatively wide and overlapping 1.0 (Table 1). There was a weak tendency that preeclampsia was associated with reduced incidence of type 1 diabetes, but this was not significant. Cesarean section was not associated with type 1 diabetes in the children (Table 1). There was a tendency that being part of a twin or higher-order multiple pregnancy was associated with a slightly decreased risk, but this was not significant, and the association was completely removed after adjustment for birth weight and other factors. Boys had an approximately $10 \%$ higher incidence rate compared with girls, regardless of adjustment for possible confounders (Table 1). As expected, the incidence of type 1 diabetes among children

Table 1. Perinatal factors and incidence of type 1 diabetes among children in Norway

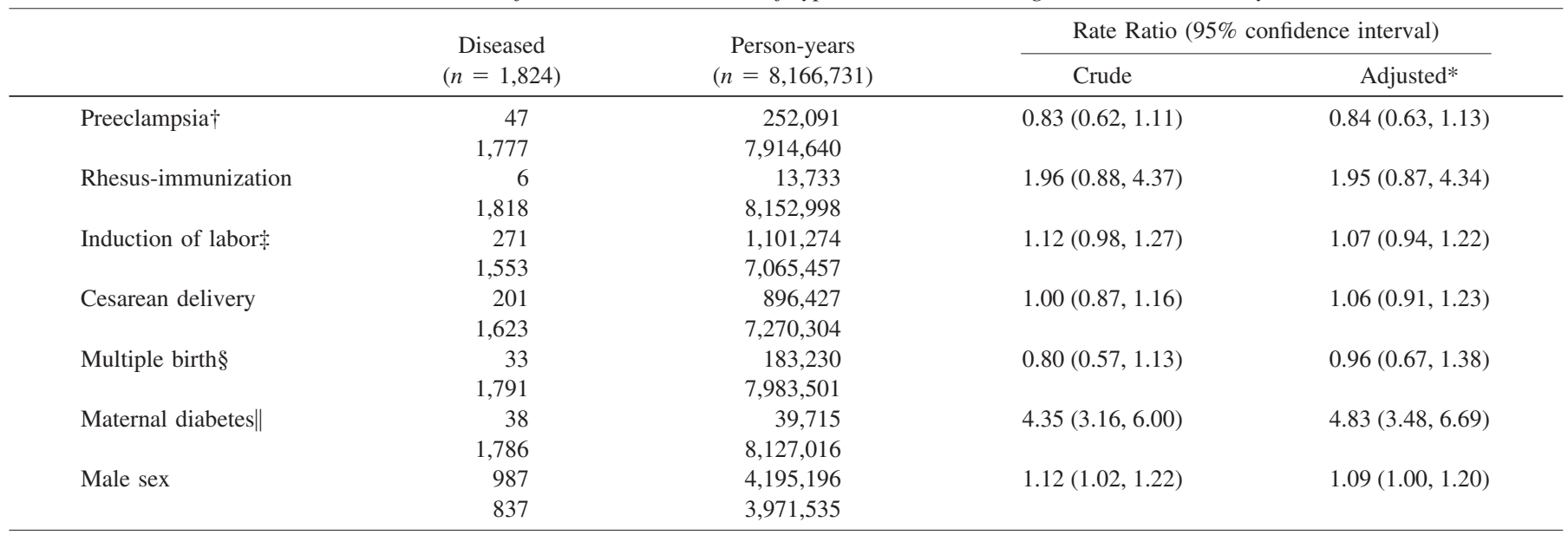

\footnotetext{
* Adjusted for sex, age, calendar period of birth, birth weight (3), maternal age, birth order, interaction between maternal age and birth order (4), and maternal diabetes.

$\dagger$ ICD-8: code 637.

$\ddagger$ By artificial tearing of membranes, use of oxcytocin, prostaglandin, or other mode of induction.

$\S$ Twin or higher-order multiple birth (all 33 cases with diabetes were twins).

$\|$ Report of any type of diabetes diagnosed before or during the index pregnancy.
} 
whose mother had diabetes diagnosed before or during pregnancy was nearly 5-fold increased compared with other children.

All results were essentially similar for different sexes, age groups, or calendar periods of birth. Furthermore, all results were essentially unaffected by adjustment for county of birth or exclusion of multiple births, births of mothers with diabetes during pregnancy, or infants with congenital malformation (data not shown).

\section{DISCUSSION}

Complications during pregnancy investigated in the present study were not significantly associated with incidence of type 1 diabetes in the children, except for the expected effect of maternal diabetes.

The very large sample size, the cohort design, and the fact that the data were based on computerized registries covering essentially the whole Norwegian population are advantages of the present study. This made it possible to adjust for a number of potential confounders simultaneously. Because the majority of births in Norway take place in the central hospital in each of the 19 counties, adjustment for county of birth should eliminate the majority of possible effects due to systematic differences in measurement protocols, completeness of reporting, or other systematic differences between the birth institutions. It is possible that some underreporting of maternal diabetes or preeclampsia could have occurred, but these are known to be rare events and the even with extreme underreporting, the proportion of misclassification among those coded as unexposed is likely to be negligibly small in our context.

Some previous studies have found a statistically significant increase in risk of diabetes associated with maternal preeclampsia $(5,7-9)$, whereas other studies have not found any significant association $(6,12)$, which is in accordance with our result. It is somewhat disturbing that the prevalence of preeclampsia among the controls in two of the previous studies was approximately $12-15 \%(7,8)$, much higher than common prevalences of about $2-5 \%$.

Blood group incompatibility has been associated with increased risk of type 1 diabetes, but Rhesus-factor incompatibility less so than $\mathrm{ABO}$ incompatibility $(5,7,9)$, if at all. Rhesus-immunization is rare, and, although an increased risk was indicated in our study, the result was not statistically significant.

Cesarean section has been associated with an approximately $20-50 \%$ increased risk of type 1 diabetes in some studies (5, 6). However, our data are in accordance with other relatively large studies, which have not found any association with cesarean section $(9,12)$. Interpretation of the inconsistent results is complicated by differences in prevalence and indications for cesarean section over time and in different countries.

It is well known that maternal diabetes is associated with increased risk of type 1 diabetes in the offspring, and previous studies have found relative risks of approximately the same magnitude as in the present study $(5,7)$. Maternal diabetes is also associated with a number of complications during pregnancy, increased fetal growth, and congenital malformations
(18). All results in the present study were essentially unchanged after adjustment for maternal diabetes or exclusion of all children whose mother had diabetes during pregnancy.

A number of previous studies indicate that some specific perinatal factors are associated with the risk of childhood-onset type 1 diabetes $(3-5,9)$. A yet-unanswered question is whether the association is explained by common genetic factors predisposing for both perinatal events and risk of type 1 diabetes. Clues to understanding the importance of intrauterine environment on risk of type 1 diabetes may be derived by comparing the concordance for type 1 diabetes among dizygotic twins with that among nontwin siblings (19). However, such a comparison rests on the assumption that being part of a twin pregnancy is itself not associated with risk of type 1 diabetes and other critical assumptions. Most previous studies have either been too small to test whether being part of a twin birth is a risk factor, or twins have been excluded from the analysis altogether. In the present study, a slightly lower incidence was indicated for twins or higher-order multiple births compared with singletons, but this was not statistically significant. It is thus not possible to exclude the possibility that this observation was due to chance, and even larger studies are necessary to draw a conclusion. Considering our previous finding of a positive association between birth weight and type 1 diabetes (3), it is tempting to speculate that the lower birth weigh of twins may explain the small, nonsignificant difference in incidence between twins and singletons, because this was completely removed after adjustment for birth weight. Intrauterine conditions for twin pairs may differ systematically from that of singletons, and nutrient availability may differ within twin pairs. Siblings have the same mother, but the intrauterine lives of siblings take place at different times during which changes in fetomaternal immune response and other changes may have occurred $(4,20)$. These factors, together with the fact that population-based twin studies with sample sizes sufficient to allow precise estimation of concordance among dizygotic twins are rare (19), make interpretation of concordance data complicated.

The basis for the different incidence of type 1 diabetes for males and females in different countries has been subject to some discussion (21), but is essentially unknown. In the present study, an approximately $10-12 \%$ higher incidence among males compared with females persisted even after adjustment for birth weight. This estimate has been notably consistent in Norway both over time (22) and over age groups (23).

\section{CONCLUSION}

In conclusion, previous indications that maternal preeclampsia and cesarean section are associated with type 1 diabetes were not supported in spite of the very large sample size of the present study. Although previous studies have shown some perinatal factors to be associated with type 1 diabetes, the majority of routinely recorded perinatal factors seem to be relatively weakly associated with the incidence of type 1 diabetes, or not at all. 


\section{APPENDIX}

Members of the Norwegian Childhood Diabetes Study Group. Henning Aabech, Fredrikstad; Helge Vogt, Nordbyhagen; Knut Dahl-Jørgensen, Oslo; Hans-Jacob Bangstad, Oslo; Geir Joner, Oslo; Kolbeinn Gudmundsson, Oslo; Olav Flesvig, Elverum; Halvor Baevre, Lillehammer; Ola Talleraas, Lillehammer; Kjell Stensvold, Drammen; Bjørn Halvorsen, Tønsberg; Kristin Hodnekvam, Porsgrunn; Ole Kr. Danielsen, Arendal; Jorunn Ulriksen, Kristiansand; Geir Stangeland, Kristiansand; John Bland, Stavanger; Dag Roness, Haugesund; Oddmund Søvik, Bergen; Per Helge Kvistad, Førde; Steinar Spangen, Ålesund; Per Eirik Hæreid, Trondheim; Sigurd Børsting, Levanger; Dag Veimo, Bodø; Harald Dramsdahl, Harstad; Kersti Thodenius, Hammerfest.

Acknowledgment. The authors thank the staff at the Medical Birth Registry for their assistance.

\section{REFERENCES}

1. Atkinson MA, Maclaren NK 1994 The pathogenesis of insulin-dependent diabetes mellitus. N Engl J Med 24:1428-1436

2. Leslie DG, Elliott RB 1994 Early environmental events as a cause of IDDM. Diabetes 43:843-850

3. Stene LC, Magnus P, Lie RT, Søvik O, Joner G, the Norwegian Childhood Diabetes Study Group 2001 Birth weight and childhood onset type 1 diabetes: population based cohort study. BMJ 322:889-892

4. Stene LC, Magnus P, Lie RT, Søvik O, Joner G, the Norwegian Childhood Diabetes Study Group 2001 Maternal and paternal age at delivery, birth order, and risk of childhood onset type 1 diabetes: population based cohort study. BMJ 323:369-371

5. Dahlquist G, Källén B 1992 Maternal-child blood group incompatibility and other perinatal events increase the risk for early-onset type 1 (insulin-dependent) diabetes mellitus. Diabetologia 35:671-675

6. Patterson CC, Carson DJ, Hadden DR, Waugh N, Cole SK 1994 A case-control investigation of perinatal risk factors for childhood IDDM in Northern Ireland and Scotland. Diabetes Care 17:376-381
7. Jones ME, Swerdlow AJ, Gill LE, Goldacre MJ 1998 Pre-natal and early life risk factors for childhood onset diabetes mellitus: a record linkage study. Int J Epidemiol 27:444-449

8. McKinney PA, Parslow R, Gurney KA, Law GR, Bodansky HJ, Williams R 1999 Perinatal and neonatal determinants of childhood type 1 diabetes: a case-control study in Yorkshire, U.K. Diabetes Care 22:928-932

9. Dahlquist G, Patterson C, Stoltesz G 1999 Perinatal risk factors for childhood type 1 diabetes in Europe: the EURODIAB substudy 2 study group. Diabetes Care 22:16981702

10. Grönlund MM, Lehtonen OP, Eerola E, Kero P 1999 Fecal microflora in healthy infants born by different methods of delivery: permanent changes in intestinal flora after cesarean delivery. J Pediatr Gastroenterol Nutr 28:19-25

11. Grönlund MM, Nuutila J, Pelto L, Lilius EM, Isolauri E, Salminen S, Kero P, Lehtonen OP 1999 Mode of delivery directs the phagocyte functions of infants for the first 6 months of life. Clin Exp Immunol 116:521-526

12. Bache I, Bock T, Vølund A, Buschard K 1999 Previous maternal abortion, longer gestation, and younger maternal age decease the risk of type 1 diabetes among male offspring. Diabetes Care 22:1063-1065

13. EURODIAB ACE Study Group 2000 Variation and trends in incidence of childhood diabetes in Europe. Lancet 355:873-876

14. Lie RT, Rasmussen S, Brunborg H, Gjessing HK, Lie-Nielsen E, Irgens LM 1998 Fetal and maternal contributions to risk of pre-eclampsia: population based study. BMJ 316:1343-1347

15. Irgens LM 2000 The medical birth registry of Norway. Epidemiological research and surveillance throughout 30 years. Acta Obstet Gynecol Scand 79:435-439

16. Stene LC 2001 Re: Implication of maternal age and birth order on type 1 diabetes; relevance in clinical practice? (eLetter). BMJ Available at: http://bmj.com/cgi/ eletters/323/7309/369\#EL4-

17. Preston DL, Lubin JH, Pierce DA, McConney ME 1993 Epicure User's Guide. Hirosoft International Corporation, Seattle

18. Persson B, Hanson U, Lunell N-O 1992 Diabetes mellitus and pregnancy. In: Alberti KG, DeFronzo RA, Keen H, Zimmet P(eds) International textbook of diabetes mellitus. John Wiley \& Sons, Chichester, pp 1085-1101

19. Kyvik KO, Green A, Beck-Nielsen H 1995 Concordance rates of insulin dependent diabetes mellitus: a population based study of young Danish twins. BMJ 311:913-917

20. Morin-Papunen L, Tiilikainen A, Hartikainen-Sorri AL 1984 Maternal HLA immunization during pregnancy: presence of anti HLA antibodies in half of multigravidous women. Med Biol 62:323-325

21. Karvonen M, Pitkaniemi M, Pitkaniemi J, Kohtamaki K, Tajima N, Tuomilehto J 1997 Sex difference in the incidence of insulin-dependent diabetes mellitus: an analysis of the recent epidemiological data. World Health Organization DIAMOND Project Group. Diabetes Metab Rev 13:275-291

22. Joner G, Søvik O 1989 Increasing incidence of diabetes mellitus in Norwegian children 0-14 years of age 1973-1982. Diabetologia 32:79-83

23. Joner G, Søvik O 1991 The incidence of type 1 (insulin-dependent) diabetes mellitus 15-29 years in Norway 1978-1982. Diabetologia 34:271-274 